E3S Web of Conferences 5,04001 (2015)

DOI: $10.1051 / \mathrm{e} 3$ sconf/ 20150504001

(C) Owned by the authors, published by EDP Sciences, 2015

\title{
On the aeroacoustic properties of a beveled plate
}

\author{
W.C.P. van der Velden ${ }^{1, a}$, A.H. van Zuijlen ${ }^{1}$, A.T. de Jong ${ }^{1}$, and H. Bijl ${ }^{1}$ \\ ${ }^{1}$ Delft University of Technology, Faculty of Aerospace Engineering, Aerodynamics Department, Kluyver- \\ weg 2, 2629HT Delft, the Netherlands
}

\begin{abstract}
The flow around a beveled flat plate model with an asymmetric 25 degrees trailing edge with three rounding radii is analyzed using a Navier-Stokes based open source software package OpenFOAM in order to predict the aeroacoustic properties of the models. A Large Eddy Simulation with a dynamic Smagorinsky and implicit model are used as closure model for the flow solver, and are compared regarding their aeroacoustic performance. Velocity coherence and pressure correlation is determined in spanwise direction. The acoustic far field spectrum is obtained by solving Curle's analogy in frequency domain as a post-processing step.
\end{abstract}

\section{Introduction}

Far field noise can be considered as one of the main design drivers for on-shore wind turbines. Strict governmental regulations limit the power production of single turbines, especially during night when background noise is less. A possible decrease of a single decibel sound pressure level would increases $20 \%$ of the annual energy production [1]. Therefore it is important to investigate possibilities to reduce noise of wind turbines, increasing the power production and reducing the cost of energy.

Previous acoustic field measurements showed that the turbulent trailing edge noise of a wind turbine blade is currently one of the most dominant noise sources on a wind turbine and therefore understanding the physics associated with the generation and propagation is of main importance for the design of more silent wind turbines [2]. This type of noise is commonly known as airfoil self-noise and originates from unsteady flow over the turbine blade. Brooks et al. [3] defined some fundamental self-noise mechanism such as the large-scale separation (deep stall), tip vortex, blunt trailing edge, laminar boundary layer and turbulent boundary layer noise. With turbulent flow, the acoustic effects depend largely on the length scale of the turbulent eddies [4]. For trailing edge noise this turbulent length scale is the boundary layer displacement thickness at the trailing edge, making the size of the eddies much smaller than the airfoil chord, thereby only effecting the local pressure fluctuations while keeping the global aerodynamic force similar. The sound of the turbulent eddies is scattered from the trailing edge towards the leading edge (i.e. in upstream direction). This typical trailing edge noise results in high-frequency edge noise.

An effective measure to investigate the local fluctuating pressure is to determine the spanwise coherence of the pressure fluctuations on the wall. Several authors, such as Amiet [5] and Howe [6] have discussed diffraction theory regarding trailing edge noise. Here, the power spectral density and

\footnotetext{
a e-mail: W.C.P.vanderVelden@TUDelft.nl
} 
spanwise coherence length of hydrodynamic pressure fluctuations were used to estimate the acoustic far field spectrum. Amiet [5] and Howe [6] assumed that the incident pressure fluctuations on the wall below the turbulent boundary convect over the trailing edge, which acts as an impedance discontinuity, where the fluctuations are scattered in the form of acoustical waves. This theory forms the basis of multiple experimental and numerical studies, such as the numerical Computational Fluid Dynamics (CFD) Large Eddy Simulation (LES) of Christophe [7] and the surface pressure measurements of Brooks and Hodgson [8].

The current study focuses on capturing trailing edge noise using a beveled flat plate model in low Mach number flow. Particularly, the spanwise coherence of both wall normal velocity and pressure fluctuations is investigated. Numerical data is obtained from a LES using the open-source package OpenFOAM in combination with two different closure models. Acoustic results are obtained using the non-homogeneous wave equation in frequency domain, in combination with wall pressure sources.

\section{Methodology}

\subsection{Governing fluid equations}

A low Mach number flow over an beveled flat plate is considered, allowing the Navier-Stokes equations to be solved. The flow is modeled as a incompressible fluid. Further, Newtonian fluid properties are assumed and gravity and any other body forces are neglected. This results in the following simplified set of equations, describing the conservation of mass and momentum:

$$
\begin{gathered}
\nabla \cdot \mathbf{u}=0, \\
\frac{\partial \mathbf{u}}{\partial t}+\nabla \cdot(\mathbf{u u})=-\frac{\nabla p}{\rho}+\nabla \cdot(v \nabla \mathbf{u}),
\end{gathered}
$$

wherein $\mathbf{u}$ are the different velocity components, $p$ is the pressure, $\rho$ the density and $v$ the kinematic viscosity. To resolve the larger eddy scales and model the smallest eddy scales, a LES methodology is applied. This methodology is known as the balanced form between completely modeling the turbulence, as done in Reynolds Averaged Navier Stokes (RANS) and completely solving all scales, as done in a Direct Numerical Simulation (DNS).

Discretization of the first time derivative is performed via a second order backward difference scheme. The velocity gradient is discretized using a second order, cell limited Gaussian linear integration with filtering for high frequency ringing. Further the velocity divergence uses a second order, Gauss linear upwind discretization while all other flow quantities are discretized using the van Leer interpolation scheme. These schemes are in general more dissipative than standard linear schemes, but with the current spatial mesh resolution, the numerical diffusion will stay sufficiently small to maintain enough resolution into the inertial range. Finally, the Laplacian is discretized using the second order, Gaussian explicit non-orthogonal correction scheme [9].

The discretized set of equations is solved in the open-source package OpenFOAM, based on the Finite Volume Method (FVM) [9]. The large time-step transient solver for incompressible flow, PIMPLEFOAM is used, which use the PIMPLE (merged PISO-SIMPLE) algorithm for obtaining the pressure. PISO is an acronym for Pressure Implicit Splitting of Operators for time dependent flows while SIMPLE stands for Semi-Implicit Method for Pressure Linked Equations which is used for steady state problems. The PISO algorithm neglects the velocity correction in the first step, but then performs one in a later stage, which leads to additional correction for the pressure [10].

The proposed subgrid scale (SGS) model in this study is the dynamic Smagorinsky model [11]. This model is an algebraic eddy viscosity SGS model in which the Smagorinsky coefficient is calculated dynamically. These coefficients are determined as part of the flow calculations, and use the 
energy content of the smallest resolved scales to locally determine the value of the closure coefficients. This implies, however, the behavior of the smallest resolved scale is analogous to that of the subgrid scales. The SGS model will be compared to an Implicit LES (ILES), where the numerical scheme itself is used such that the inviscid energy cascade through the inertial range is captured accurately and the inherent numerical dissipation emulates the effect of the dynamics beyond the grid-scale cut-off [12].

\subsection{Recycled inflow}

To simulate a fully turbulent boundary layer on a flat plate within current computational capacity, a recycling method is used. The main idea behind the recycling and rescaling inflow modeling approach is to extract data at a station downstream from the inflow, and rescale it to account for boundary layer growth. In the approach by Lund [13], the flow at the extraction station is averaged in spanwise direction and in time, to allow the decomposition of the flow field in a mean and fluctuating part. The mean velocities and fluctuations are then rescaled according to the law of the wall in the inner region and the defect law in the outer region, and blended together using a weighted average of the inner and outer profiles:

$$
\left(u_{i}\right)_{\text {in }}=\left\{\left(U_{i}\right)_{\text {in }}^{\text {inner }}+\left(u_{i}^{\prime}\right)_{\text {in }}^{\text {inner }}\right\}\left[1-W\left(\eta_{\text {in }}\right)\right]+\left\{\left(U_{i}\right)_{\text {in }}^{\text {outer }}+\left(u_{i}^{\prime}\right)_{\text {in }}^{\text {outer }}\right\}\left[W\left(\eta_{\text {in }}\right)\right],
$$

with the weighting function defined as:

$$
W(\eta)=\frac{1}{2}\left\{1+\frac{1}{\tanh (\alpha)} \tanh \left[\frac{\alpha(\eta-b)}{(1-2 b) \eta+b}\right]\right\},
$$

wherein $\eta=y / \delta$ indicates the outer coordinate scaling and $\alpha=4$ and $b=0.2$ are prescribed constants [13].

\subsection{Coherence}

For determination of the coherence length, first the coherence function should be evaluated. The coherence function is the auto-power and cross-power density of the signals, where $\Phi\left(\omega, z_{1}, z_{2}\right)$ denotes the cross-power spectral density between two points along a given dimensional line $\Delta z=z_{2}-z_{1}$, with $\omega$ defined as the angular frequency:

$$
\gamma^{2}(\omega, \Delta z)=\frac{\left|\Phi\left(\omega, z_{1}, z_{2}\right)\right|^{2}}{\left|\Phi\left(\omega, z_{1}, z_{1}\right)\right|\left|\Phi\left(\omega, z_{2}, z_{2}\right)\right|} .
$$

This representation is valid for the case that the flow statistics are homogeneously distributed along the spatial dimension, stationary in time and for an infinite observation period. For a flat plate the first criteria is fulfilled when considering the spanwise direction, and with restriction to very short separations, also for the streamwise direction. By definition, the coherence length is related to the integral of coherence function over the spatial separation $\Delta z$ and therefore reduces to a function of frequency only:

$$
l_{z}(\omega)=\lim _{L \rightarrow \infty} \int_{0}^{L} \gamma(\omega, \Delta z) d \Delta z
$$




\subsection{Acoustics}

Within the field of Computational Aero-Acoustics (CAA) a distinction is made between direct and hybrid methods. Direct CAA methods solve the full compressible flow equations for determining both the hydrodynamic and acoustic pressure fluctuations. The domain covers both the flow field and at least the source and near acoustic field. Due to the high computational cost originating from the large scale separation of hydrodynamic fluid and acoustic pressure fluctuations, a direct calculation is restricted to simple geometries and low and moderate Reynolds numbers. In a hybrid method the flow and acoustic field are calculated separately, so that the numerical method can be optimized for the physics to be solved.

In this study the acoustic field is based on the non-homogeneous wave equation from Lighthill [14], but extended with surface sources in presence of turbulent fluctuations near a solid body [15]. Curle's analogy is translated to frequency domain by Lockard [16] and by assuming subsonic rectilinear motion of all acoustic sources, an efficient and easy implementable form of Curle's analogy is obtained which determines the far field noise from non-linear nearfield flow quantities:

$$
H(f) c_{0}^{2} \rho^{\prime}(\mathbf{y}, \omega)=-\int_{f=0} F_{i}(\xi, \omega) \frac{\partial G(\mathbf{y} ; \xi)}{\partial y_{i}} d s,
$$

wherein $G$ indicates the three dimensional free-field Green function and $F$ includes the source term from the CFD simulaton:

$$
F_{i}=p \hat{n}_{i},
$$

with $\hat{n}_{i}$ being the outward pointing normal vector on the surface. Further, $\xi$ denotes the three dimensional source coordinates while $\mathbf{y}$ indicates the current observer position.

\subsection{Model set-up}

Using the prescribed methodology, the flow around a $360 \mathrm{~mm}$ length $(c), 20 \mathrm{~mm}$ thick $(t)$ and $20 \mathrm{~mm}$ $\operatorname{span}(s)$ beveled flat plate with an asymmetric 25 degrees trailing edge with $0 t$, $4 t$, and $10 t$ rounding radii under a Reynolds numbers of $2.68 \cdot 10^{5}$ is analyzed. The domain stretches for $4 t$ in both wall normal directions and extends $0.5 c$ in the wake region. Mesh resolution in streamwise, wall-normal and spanwise direction is set to $X^{+}=50, Y^{+}=1$ and $Z^{+}=10$ respectively, corresponding to $7.5 \cdot 10^{6}$ cells with an $Y^{+}$of $3.0 \cdot 10^{-5} \mathrm{~m}$. An overview of the structured mesh is depicted in Fig. 1.

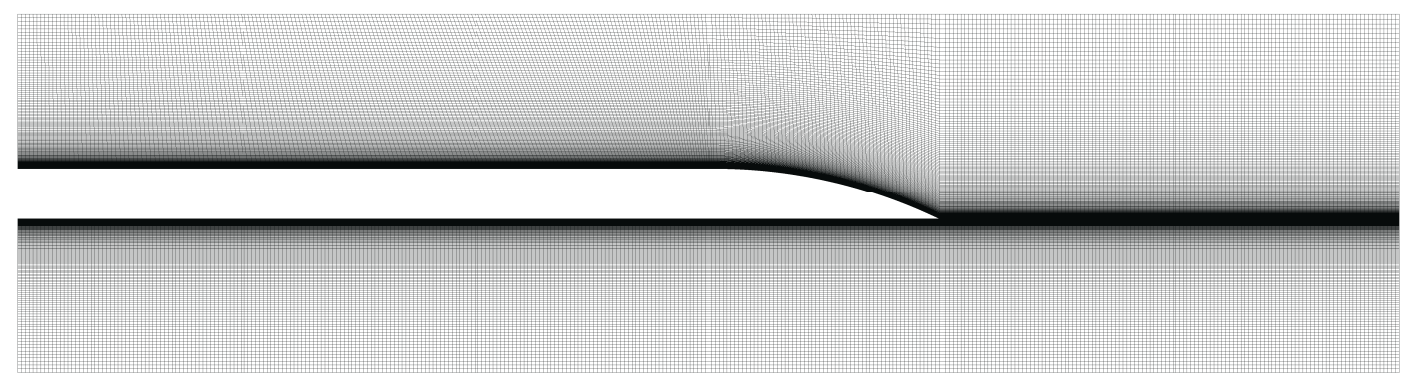

Figure 1. Snapshot of the mesh of the $10 \mathrm{t}$ beveled flat plate

The velocity inflow is prescribed according to the method discussed in section 2.2 , and results in a turbulent boundary layer with a boundary layer thickness, momentum thickness, displacement 
thickness and shape factor of $6.5 \mathrm{~mm}, 0.7 \mathrm{~mm}, 1.0 \mathrm{~mm}$ and 1.45 respectively at the start of the rounding radii of the beveled plate. The velocity outlet is modeled using a Neumann condition. A noslip condition for the flat plate wall is used and top and bottom are modeled similar as the outflow. The front and back patches are cyclic, which simulates an infinite span condition. Regarding the boundary condition for the pressure, zero gradient conditions are used for inlet, outlet and wall. The top and bottom are modeled using a Dirichlet condition, imposing the atmospheric free stream condition on the edges.

\section{Results}

\subsection{Velocity}

The instantaneous streamwise velocity, illustrated in Fig. 2 were obtained from the CFD simulation methodology described in previous section. Sampling is done at $27 \cdot 10^{3} \mathrm{~Hz}$, with a total sampling time of $0.15 \mathrm{~s}$, resulting in four complete flow passes. The general trends of a beveled trailing edge begins with an upstream turbulent boundary layer on the upper side separating due to the adverse pressure gradient that develops into a shear layer. The lower boundary layer remained relatively undisturbed before separating at the sharp trailing edge. The low momentum fluid contained in the separated region was bound by high momentum fluid on either side, generating two regions of shear in the near wake. Flow reversal clearly demonstrates the separated flow regions. The $0 t$ model shows boundary layer separation over the wedge region with an extensive zone of recirculating motion whereas in the $10 t$ model, no flow separation occur. This difference can be attributed to the less intense adverse pressure gradient due to the large surface curvature. The $4 t$ model clearly shows the four different regions $[4,17,18]$ : the upper shear layer region, the separation bubble or recirculation zone, the lower shear layer region and the wake region.
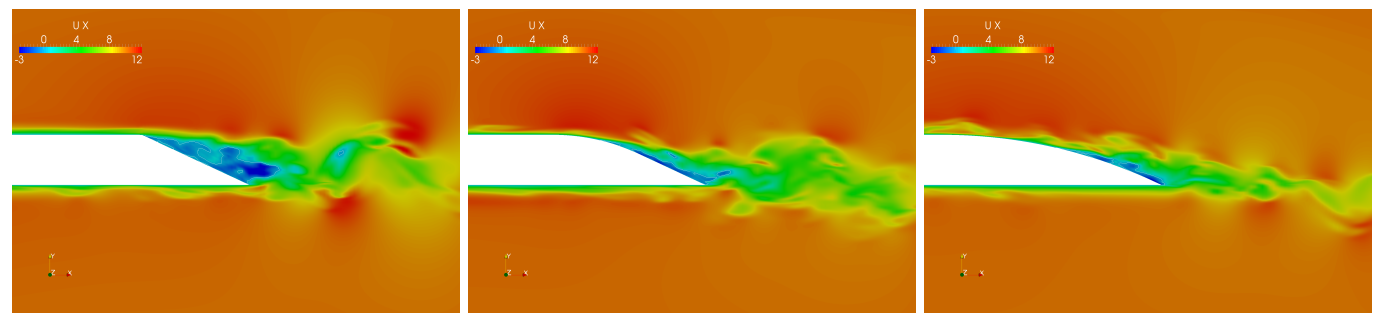

Figure 2. Contours of instantaneous streamwise velocity for $0 t, 4 t$ and $10 t$

To further discuss velocity statistics, the mean and rms along the streamwise stations at $x / t=-4.625(A),-3.125(B),-2.125(C),-1.625(D),-1.125(E),-0.625(F), 0(G)$ in a coordinate system originating from the trailing edge are depicted in Fig. 3. Both dynamic Smagorinsky and implicit closure model are compared. In general good agreement is found for the first three stations for both SGS models. The turbulent boundary layer, the stronger adverse pressure gradient regions and unsteady separated region, discussed in previous paragraph, are clearly visualized. The near-wall peaks in the rms horizontal velocity are also clearly illustrated, which is common to exist in turbulent boundary layers. It can be observed that the flow profile changes as the bevel radius of curvature increases because the corresponding adverse pressure gradient also decreases. A larger discrepancy between both closure models is found downstream the $10 t$ model. This variation can be assigned to the nature of separation, as well as the way numerical dissipation occurs in the implicit model. For the $0 t$ model, 
separation is geometry based and appears at the same location for both closure models, whereas in the $10 t$ case, separation is caused by the incoming turbulent boundary layer and will therefore vary in both closure models simulations because of the difference in modeling the dissipation. When the wall normal cell size increases, more dissipation in ILES models occur, reducing the rms velocity.
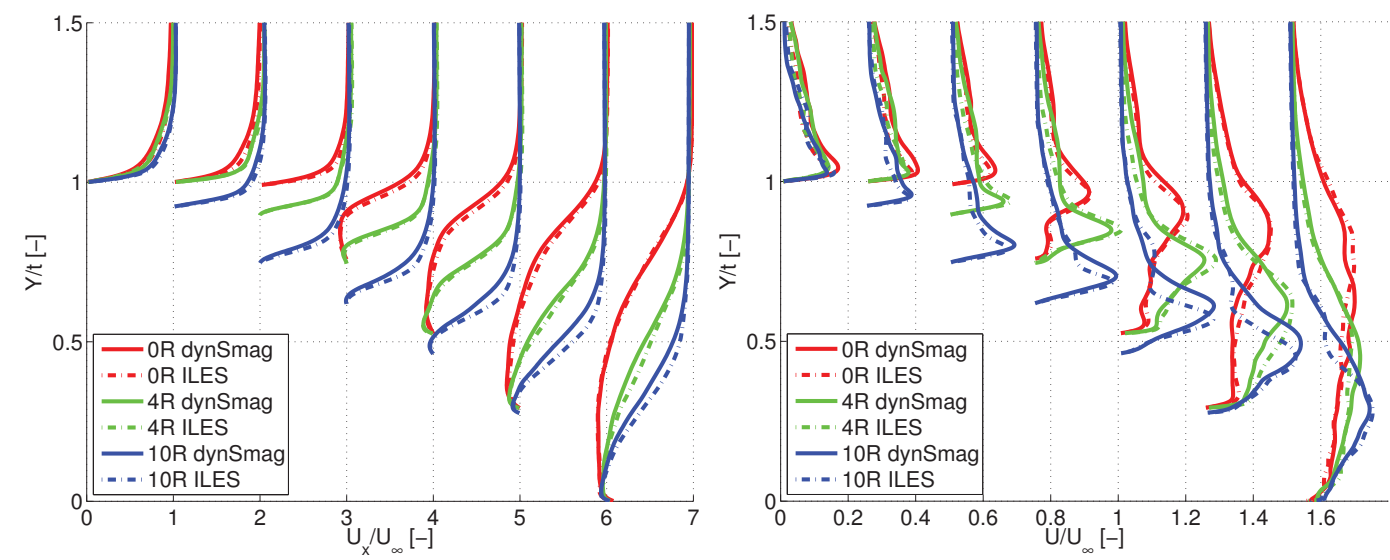

Figure 3. Profile of the normalized mean (left) and rms (right) horizontal velocity as a function of vertical distance, individual profiles are separated by a horizontal offset of 1 and 0.25 on the left and right side respectively

A closer, qualitatively look at the unsteady organization and evolution of coherent structures within the turbulent boundary layer is further investigated using Fig. 4, where the second invariant of the velocity gradient tensor $Q$ is plotted. The interaction between low speed streaks (blue) and vortical structures (red) are shown by means of hairpin packets, full hairpins, legs and cane vortices [19].

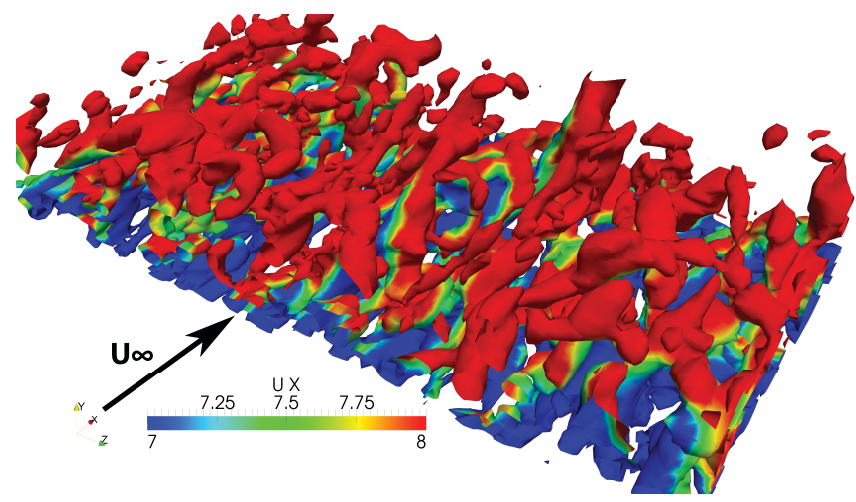

Figure 4. Instantaneous visualization of a portion of the turbulent boundary layer upstream of the beveled flat plate using isosurfaces of the second invariant of the velocity gradient tensor, $Q=0.2 \cdot 10^{6}$ of the ILES model, colored by the streamwise velocity

The ILES method is further investigated by discussing the spanwise roll-up of vortices along the sharp beveled trailing edge. Therefore, the spanwise coherence of wall normal velocity fluctuations 
at different stations along the core of the shear layer is illustrated in Fig. 5. Here, a contour plot of the coherence function is depicted, which is, as shown in Eq. 5, dependent on a spatial coordinate (spanwise in this specific case) and on the frequency. The coherence is determined according to the method described in Sec. 2.3. Clearly, there is one specific range of Strouhal numbers, which characterizes the coherent spanwise structures. Further downstream, the frequency range increases when turbulent structures are merging and separating. The overall coherence decreases due to this mixing shear layer.
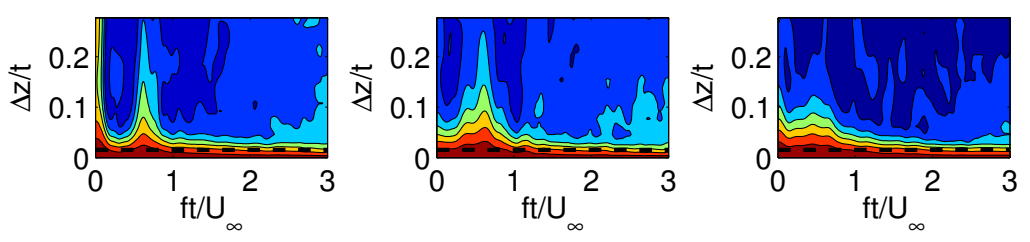

Figure 5. Spanwise coherence of wall normal velocity fluctuations at different stations along a line in the core of the shear layer for $0 t$. From left to right: $d x=0,9$ and 18

\subsection{Pressure}

The temporal variations of wall-pressure fluctuations, important to analyze the acoustic far field spectrum, are exemplified in Fig.6. The lines are obtained at the stations introduced before in Fig. 3, walking from the upstream locations at the bottom of the figure towards the trailing edge (top). In general, at the upstream stations, in the attached turbulent boundary layer region, pressure signals consist predominantly of high-frequency fluctuations associated with small-scale eddies. The amplitude of the oscillations is decreased in the in favorable pressure gradient regions for the $4 t$ and $10 t$ cases and increased in the adverse pressure gradient region. After the separation of the boundary layer (further downstream stations), the high frequency content has vanished and the surface pressure is characterized by lower frequency and higher amplitude oscillations, espically at the $4 t$ model. This is likely caused by the unsteady separation. At the trailing edge (top line), some of the higher frequency content reappear because of the contribution of the attached turbulent boundary layer on the lower side of the beveled plate, while the unsteady flow on the upper side accounts for the large amplitude, lower frequency content.

Analyzing the spectral content of the wall pressure further, space-time correlation plots of the upper surface pressure fluctuations as a function of temporal and spanwise separations are shown in Fig. 7. Before the turbulent boundary layer becomes separated, small variations of the spanwise spatial and termporal scales are present underneath the boundary layer. After separation, larger spatial and temporal scales occur. The wall pressure fluctuations inside the separated region are now dominated by large coherent structures. The smaller scales are swepped away from the wall and do not appear on the spectrum anymore. Further it can be noted that the space-time correlation plots show insufficient drops at maximum spanwise separation at the downstream stations, suggesting that the spanwise domain is insufficient large to allow for the development of fully three dimensional large-scale flow structures. 

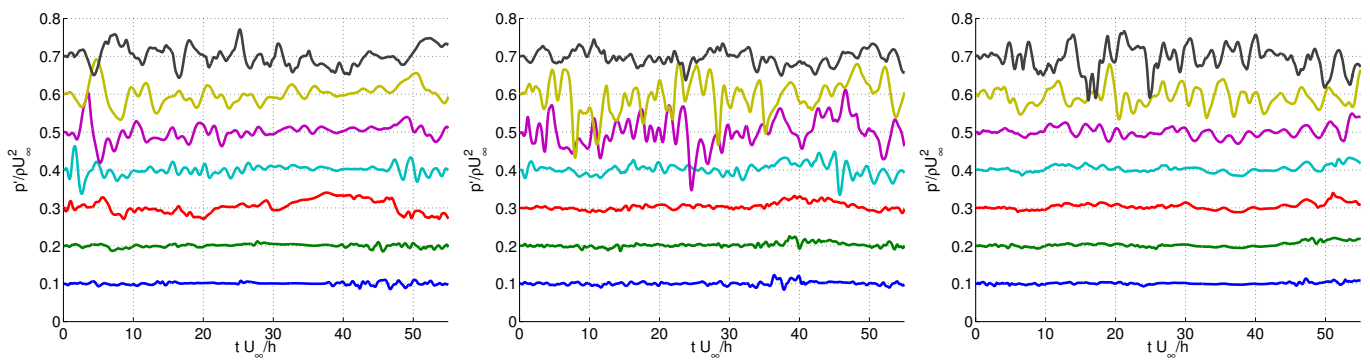

Figure 6. Time history of surface pressure fluctuations at streamwise stations, upstream bottom, downstream top at a fixed spanwise coordinate; the individual curves are separated by a vertical offset. From left to right: $0 t, 4 t$, $10 t$
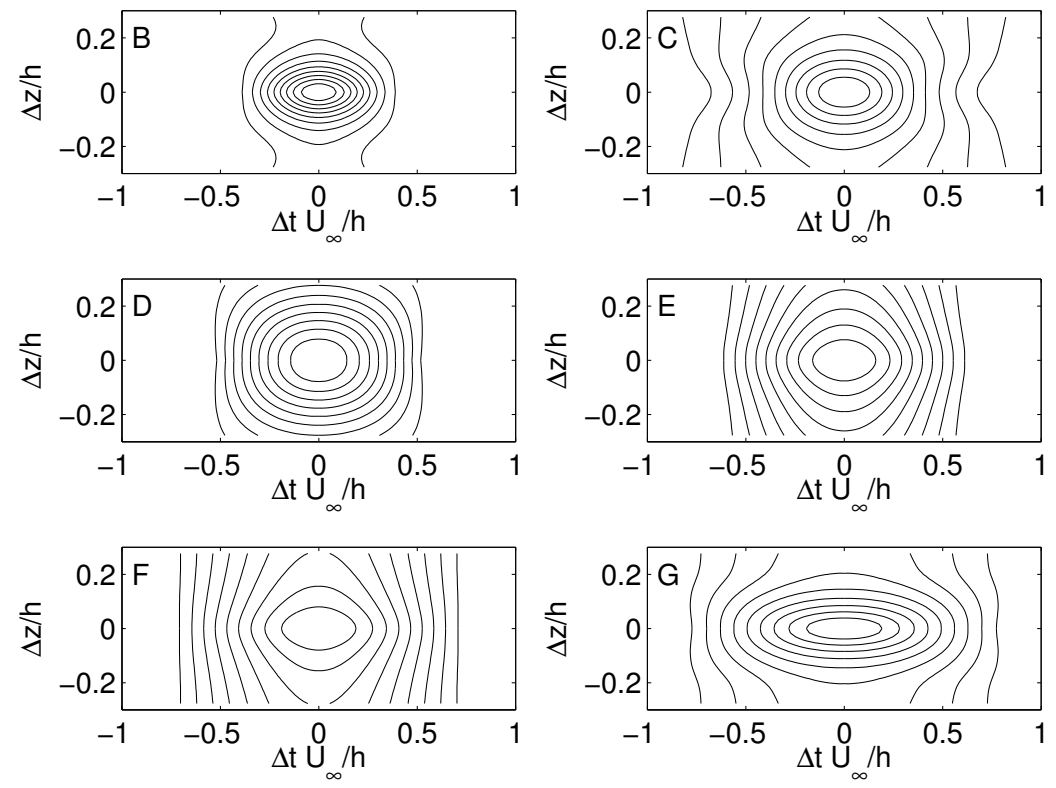

Figure 7. Contours of space time correlation of the upper-surface pressure fluctuations as a function of spanwise and temporal separations at the indicated stations; contour values are from 0.1 to 0.9 , with increment 0.1

\subsection{Acoustics}

The acoustic far field spectrum, determined with Curle's analogy discussed in Sec. 2.4, is shown in Fig. 8. The one-third octave Sound Pressure Level (SPL) at a distance of $10 c, 90$ degrees above the trailing edge is illustrated for each model with the following SPL correction factor:

$$
S P L_{1 / 3}^{c o r r}=S P L_{1 / 3}-50 \log _{10}(M)-10 \log _{10}\left(\frac{\delta^{*} \cdot s}{R^{2}}\right)
$$

with $M, \delta^{*}, s$ and $R$ indicating the Mach number, displacement thickness, span and observer radius dependence. As expected, the geometry triggered boundary layer separation generates a clear peak 
in the frequency spectrum plot, as can be seen from the 0t line. When separation moves further downstream ( $4 t$ model), the separation length scale decreases, thereby shifting the peak to higher frequencies. At the $10 t$ model, nearly separation occurs and separation is determined by the nature of the turbulent boundary layer. The boundary layer, which consists of many different coherent length scales, nicely convects over the trailing edge, resulting in a broadband noise spectrum. The dynamic Smagorinsky and ILES closure models show similar results for both $0 t$ and $4 t$ models, but a larger discrepancy at the $10 t$ model. Especially with the most favorable pressure gradient model, where separation is not geometry based but more a function of many characteristics inside the turbulent boundary layer, correct modeling is essential.

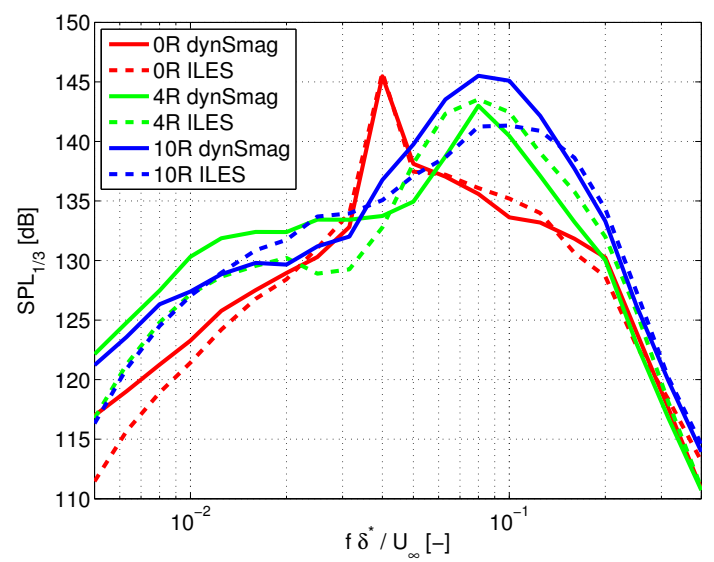

Figure 8. Frequency spectra of the far field noise at $R=10 c, 90$ degrees above the trailing edge

Instead of looking 90 degrees above the trailing edge only, an entire directivity analysis on 72 angles is performed in Fig. 9 for three different frequency ranges. The general pattern, lobes on pressure and suction side respectively are in line with literature [17]. Both lobes are rotated in clockwise direction. This rotation is due to the effective chord of the relatively thick beveled part of the plate, now having the lobes perpendicular on the chord. Higher Overall Sound Pressure Levels (OASPL) are observed at Strouhal numbers of $0.05-0.2$, with the $10 t$ model being the most effective sound emitting model. At lower Strouhal numbers, the $0 t$ and $4 t$ model are more dominantly, mainly because of the tonal noise peak associated with a larger separation length scale.

\section{Conclusion}

The incompressible Navier-Stokes equations, together with two SGS models are solved around three beveled trailing edge models using the open source package OpenFOAM to investigate the aeroacoustic behavior of the models. The sharp beveled model shows a separation point right at the kink, while the $4 t$ model shows unsteady separation along the rounding radii and at the $10 t$ model separation and reattachment occurs due to the favorable pressure gradient. The implicit closure model deviates most at the $10 t$ model at a small distance from the wall, probably due to the larger dissipation at larger cells positioned away from the boundary, making it hard to model boundary layer based separation. Close to the wall however, a good resemblance with the dynamic Smagorinsky model is found; the turbulent boundary layer and turbulent structures such as hairpin packages and low streaks regions are clearly captured. 

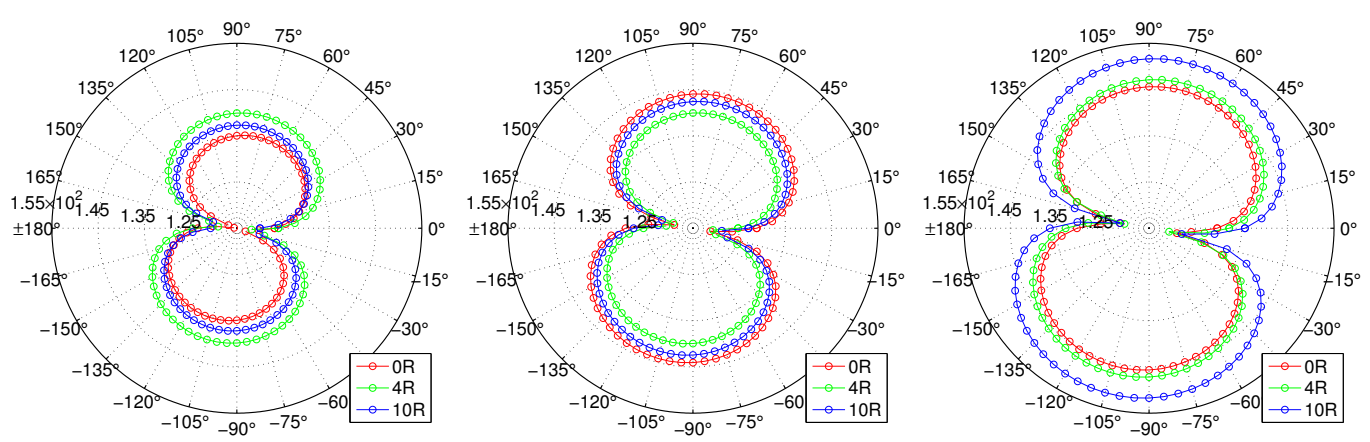

Figure 9. Directivity plot of the corrected Overall Sound Pressure Level (OASPL) for different Strouhal numbers; $0.005-0.025$ (left), $0.025-0.05$ (middle) and $0.05-0.2$ (right)

The spanwise coherence of wall normal velocity fluctuations at the 0t model shows a clear distinctive peak due to spanwise roll-up of coherent structures. Further downstream the wake, in the jet shear layer, this peak transfers to a more broadband spectrum, separating and merging coherent structures.

The pressure fluctuations below the turbulent boundary layer, on the wall, are characterized by high frequency, low amplitude oscillations. Further downstream, where the boundary layer separates this behavior changes to lower frequency, higher amplitude oscillations, likely to occur due to unsteady, shifting, separation. Space-time correlation plots at the various stations show low temporal coherence upstream and high temporal coherence downstream. Spanwise correlation insufficiently drops, suggesting a too small spanwise domain to account for large three-dimensional spanwise structures.

The acoustic far field spectrum, solved using Curle's analogy in frequency domain, shows clear tonal noise for the 0t fixed separation model, while broadband noise is observed for the 10t model, due to a turbulent boundary layer convecting over the entire trailing edge span. A slightly rotated lobe behavior is observed for the OASPL, in line with literature regarding the directivity effects of trailing edge noise.

\section{References}

[1] S. Oerlemans, P. Fuglsang, Siemens AG (2012)

[2] S. Oerlemans, P. Sijtsma, B.M. Lopez, Journal of Sound and Vibration 299, 869 (2007)

[3] T. Brooks, D. Pope, M. Marcolini, Tech. rep., NASA Reference Publication 1218 (1989)

[4] W. Blake, Mechanics of flow-induced sound and vibration, volumes I and II (Academic Press, 1986)

[5] R. Amiet, Journal of Sound and Vibration 47, 387 (1976)

[6] M. Howe, Journal of Sound and Vibration 225, 211 (1999)

[7] J. Christophe, Ph.D. thesis, Université Libre de Bruxelles (2011)

[8] T. Brooks, T. Hodgson, Journal of Sound and Vibration 78, 69 (1981)

[9] OpenFOAM, OpenFOAM: The Open Source CFD Toolbox Programmer's Guide (ESI-Group, 2012)

[10] R. Issa, Journal of Computational Physics 62, 40 (1986)

[11] M. Germano, U. Piomelli, P. Moin, W. Cabot, Physics of Fluids 3, 1760 (1991) 
[12] J. Boris, Fluid Dynamics Research 10, 199 (1992)

[13] T. Lund, X. Wu, K. Squires, International Journal of Computational Physics 140, 233 (1998)

[14] M. Lighthill, Proceedings of the Royal Society of London 211, 564 (1952)

[15] N. Curle, Proceedings of the Royal Society of London 231, 505 (1955)

[16] D. Lockard, Journal of Sound and Vibration 229, 897 (2000)

[17] M. Wang, Center for Turbulence Research, Annual Research Briefs pp. 91-106 (1998)

[18] D. Shannon, S. Morris, Experiments in Fluids 41, 777 (2006)

[19] S. Ghaemi, F. Scarano, Journal of Fluid Mechanics 689, 317 (2011) 